Bond University

Research Repository

\title{
Quantification of Competitive Game Demands of NCAA Division I College Football Players Using Global Positioning Systems
}

\author{
Wellman, Aaron D.; Coad, Sam C.; Goulet, Grant C.; McLellan, Christopher P. \\ Published in: \\ Journal of Strength and Conditioning Research
}

DOI:

10.1519/JSC.0000000000001206

Licence:

Other

Link to output in Bond University research repository.

Recommended citation(APA):

Wellman, A. D., Coad, S. C., Goulet, G. C., \& McLellan, C. P. (2016). Quantification of Competitive Game Demands of NCAA Division I College Football Players Using Global Positioning Systems. Journal of Strength and Conditioning Research, 30(1), 11-19. https://doi.org/10.1519/JSC.0000000000001206

\section{General rights}

Copyright and moral rights for the publications made accessible in the public portal are retained by the authors and/or other copyright owners and it is a condition of accessing publications that users recognise and abide by the legal requirements associated with these rights.

For more information, or if you believe that this document breaches copyright, please contact the Bond University research repository coordinator. 
QUANTIFICATION OF COMEPTITIVE GAME DEMANDS OF NCAA DIVISION I COLLEGE FOOTBALL PLAYERS USING GLOBAL POSITIONING SYSTEMS

${ }^{1}$ Faculty of Health Sciences and Medicine, Bond University, Queensland, Australia.

${ }^{2}$ University of Michigan, School of Kinesiology, Ann Arbor, MI.

\section{ABSTRACT}

The aim of the present study was to examine the competitive physiological movement demands of NCAA Division I college football players using portable global positioning system (GPS) technology during games, and to examine positional groups within offensive and defensive teams, to determine if a player's physiological requirements during games are influenced by playing position. Thirty-three National Collegiate Athletic Association (NCAA) Division I Football Bowl Subdivision football players were monitored using GPS receivers with integrated accelerometers (GPSports, Canberra, Australia) during 12 regular season games throughout the 2014 season. Individual datasets $(n=295)$ from players were divided into offensive and defensive teams, and 
21 subsequent position groups. Movement profile characteristics including total, low-,

22 moderate-, high-intensity and sprint running distances (m), sprint counts, and acceleration and deceleration efforts, were assessed during games. A one-way ANOVA and post-hoc Bonferroni statistical analysis were used to determine differences in movement profiles between each position group within offensive and defensive teams. For both offensive and defensive teams, significant $(p<0.05)$ differences exist between positional groups for game physical performance requirements. The results of the present study identified that wide receivers (WR) and defensive backs (DB) completed significantly $(p<0.05)$ greater total distance, high-intensity running, sprint distance, and high-intensity acceleration and deceleration efforts compared to their respective offensive and defensive positional groups. Data from the present study provide novel quantification of position specific physical demands of college football games and support the use of position-specific training in the preparation of NCAA Division I college football players for competition.

Key Words: GPS, monitoring, American football

\section{INTRODUCTION}

American football is a field-based team sport requiring high levels of muscular strength, power, speed and agility, and is characterized by intense collisions and repeated highintensity movements (27). American football games are intermittent in nature involving short-duration high-intensity bouts of exercise which incorporate movements such as 
44 sprinting, backpedaling, accelerating, decelerating, and physical collisions, separated by transient periods of low-intensity recovery between plays (12). During the in-season period of competition, players competing in NCAA Division I college football are

47 required to participate in twelve regular season games on a consecutive weekly basis. Few studies have investigated $(12,26)$ the demands of NCAA Division I football games and as such, the movement characteristics of competition in college football players remain ambiguous. While research $(12,26)$ has provided a rudimentary description of exercise to rest ratios encountered during NCAA Division I college football games, a

52 more detailed assessment of position-specific movement demands during competition provides novel insight to improve our understanding of the demands of competition and

54 enable increased scope for position-specific training and conditioning programs to 55 optimize on-field performance.

57 The development of global positioning system (GPS) technology with integrated tri-axial accelerometers have allowed the physiological demands of training and competition in

59 contact team sport to be quantified by tracking the movement of players $(2,10,32)$. Improvements in GPS technology have subsequently resulted in enhancements in

61 accuracy (13), and the validity and reliability of GPS to determine the movement

62 demands of team sports is well established $(6,14,15,30)$. The quantification of team-

63 sport competition demands using GPS technology has been reported in sports similar in

64 nature to American football, including rugby league $(2,10,24)$, rugby sevens $(11)$,

65 Australian football league (AFL) $(18,29,31)$, and rugby union $(7,21)$. Further

66 substantiating the use of GPS technology to accurately determine position-specific 
67 demands of team sport, Boyd et. al. (4) demonstrated the capacity of GPS units with 82 activities (8). integrated accelerometry to differentiate between training drills and competitive games, and discriminate between players competing in elite and sub-elite team-sport competitions. Although GPS technology is widely used in team sports for analysis of game and training movement demands, current literature on the movement profile characteristics of American football players is limited (8).

DeMartini et. al. (8) reported movement profile characteristics associated with preseason training sessions in NCAA Division I college football by examining the physical demands of Division I college football players during nine pre-season practices over the course of eight days, utilizing GPS to evaluate total distance covered and running velocity characteristics. The main findings reported by DeMartini et. al. (8) were that non-linemen covered greater total distance and sprint distance than linemen, who covered greater distance at slower speeds. To date, ambiguity remains regarding the demands of in-season NCAA Division I college football games and team training In American football each position group has distinct physiologic and biomechanical 85 demands associated with specific technical and tactical requirements (16), however uncertainty exists regarding the position-specific movement demands of NCAA football

87 competition. Given the widespread inclusion of GPS technology in collegiate American 88 football programs, a detailed assessment of competitive movement profile 
characteristics will provide sports performance specialists with quantified information on game demands. A more comprehensive understanding of the demands of NCAA football competition will augment our understanding of the position-specific movement demands of NCAA college football players, and allow sport coaches to individualize training programs that replicate the demands of American football games.

The aim of the present study was to 1) examine the competitive physiological movement demands of NCAA Division I college football players using portable global positioning system (GPS) technology during games, and 2) to examine positional groups within offensive and defensive teams, to determine if a player's physiological requirements during games are influenced by playing position. We hypothesized that there will be substantial positional differences in movement demands of NCAA Division I college football players during games. Data obtained will provide scope for performance coaches seeking to optimize position-specific training regimens.

\section{METHODS}

\section{EXPERIMENTAL APPROACH TO THE PROBLEM}

Portable GPS and integrated tri-axial accelerometry technology was used in the present study to quantify the position-specific movement characteristics of NCAA Division I 
110 college football games. The GPS movement profile data was collected during twelve

111 regular season NCAA Division I college football games. All games were 60-minutes in

112 duration, comprised of four 15-minute quarters, each followed by a brief recovery

113 period, and played outdoors between the hours of 12:00 and 21:00 over a period of

114 twelve to thirteen weeks from September to November. All participants were required to

115 participate in a minimum of $75 \%$ of the total offensive or defensive plays for the GPS

116 datasets to be included in the present study. Each individual GPS dataset was

117 characterized as constituting either offensive or defensive team performance, and

118 subsequently divided into specific positional groups for the offense that included wide

119 receivers (WR), quarterbacks (QB), running backs (RB), tight ends (TE), offensive

120 linemen (OL), and for the defense that included defensive backs (DB), linebackers (LB),

121 defensive ends (DE) and defensive tackles (DL).

123 SUBJECTS

125 Thirty-three National Collegiate Athletic Association (NCAA) Division I Football Bowl 126 Subdivision (FBS) football players (age $20.7 \pm 1.0$ years; height $188.6 \pm 7.2 \mathrm{~cm}$; and 127 mass $106.7 \pm 19.6 \mathrm{~kg}$ ) participated in the present study. The heights and weights for 128 each position group are expressed as means \pm standard deviation and presented in 129 Table 1. All subjects were collegiate athletes whom had been selected to participate in 130 the football program eight months prior to the commencement of the study. All

131 participants in the present study took part in the teams' off-season physical 
132 development training program that included a full-body strength and power training

133 program and specific skills and conditioning sessions designed to simulate the demands

134 of NCAA Division I college football competition. The present study comprises statistical

135 analysis of data collected as part of the day to day student athlete monitoring and

136 testing procedures within the university's football program. Researchers were provided

137 with de-identified GPS datasets from twelve regular season games for analysis. De-

138 identified data included participant playing position for the purposes of position-specific

139 data analysis. Ethical approval was obtained from the the university's human research

140 ethics committee.

\section{PROCEDURES}

Global Positioning System Units. The present study used commercially available GPS

147 receivers (SPI HPU, GPSports, Canberra, Australia) which operated in a non-differential

148 mode at a sampling frequency of $15 \mathrm{~Hz}$. The GPS receivers also contain integrated tri-

149 axial accelerometers (IA), which operated at $100 \mathrm{~Hz}$ and assessed the frequency and

150 magnitude of full-body acceleration $\left(\mathrm{m} \cdot\right.$ second $\left.^{-2}\right)$ in three dimensions, namely, anterior-

151 posterior, mediolateral, and vertical $(17,22)$. Subjects had previously worn GPS

152 receivers in outdoor training sessions that included football-specific running, and skill-

153 related and game-simulated contact activities during a three week pre-season training 
154 period. Prior to the commencement of each game, GPS receivers were placed outside

155 for 15 minutes to acquire a satellite signal, after which, receivers were placed in a 156 custom designed pocket attached to the shoulder pads of the subjects. Shoulder pads 157 were custom-fit for each individual, thereby minimizing movement of the pads during 158 competition. The GPS receivers used in the present study (66 g; $74 \mathrm{~mm} \times 42 \mathrm{~mm} \times 16$ $159 \mathrm{~mm}$ ) were positioned in the center of the upper back, slightly superior to the scapulae. 160 Subjects were outfitted with the same GPS receiver for each of the twelve games. 161 Following the completion of games, GPS receivers were removed from the shoulder 162 pads, and subsequently downloaded to a computer for analysis utilizing commercially 163 available software (Team AMS, GPSports, Canberra, Australia). The validity and 164 reliability of GPS to measure distance and velocity during high-intensity exercise that 165 characterizes contact and noncontact team sports have been reported $(3,9,14,25)$. 166 Johnston et. al. (14) have demonstrated GPS receivers utilized in the present study to 167 be valid for measuring total distance and average peak speed in a team sport simulation 168 circuit, with intraclass correlation values of interunit reliability reported to be 0.94 for 169 high speed running $\left(14.00-19.99 \mathrm{~km} \cdot \mathrm{h}^{-1}\right)$ distance, 0.81 for very high speed running (> $17020.00 \mathrm{~km} \cdot \mathrm{h}^{-1}$ ) distance, -0.20 for total distance, and -0.14 for peak speed.

172 Data provided from GPS receivers were assessed as movement profile variables 173 including total, low-intensity, moderate-intensity, high-intensity and sprint distances (m), 174 max velocity achieved $(\mathrm{km} / \mathrm{h})$, and counts of sprint, acceleration and deceleration 175 efforts. Classifications of parameters of movement profile variables are described 176 below and presented in Table 2. Each of the GPS variables measured in the present 
177 study was calculated using commercially available software (Team AMS, GPSports, 178 Canberra, Australia).

Movement Profile Classification. Movement profile classifications have been described

181 for game analysis in similar contact team sports $(19,20,23,24)$, however the

182 classification profile utilized in the present study was devised for American football

183 players. Each movement classification was coded as one of four speeds of locomotion

184 (Table 2). Low-intensity movements, such as standing, walking and light jogging, were

185 considered to be $0-10 \mathrm{~km} \cdot \mathrm{h}^{-1}$, moderate-intensity movements, such as a cruising jog,

186 were considered to be $10.1-16.0 \mathrm{~km} \cdot \mathrm{h}^{-1}$, high-intensity movements, such as fast jog or

187 striding, were classified as $16.1-23.0 \mathrm{~km} \cdot \mathrm{h}^{-1}$, and sprinting or maximal effort

188 movements were classified as exceeding $23.0 \mathrm{~km} \cdot \mathrm{h}-1$. Short duration high-intensity

189 movement efforts, or measures of acceleration and deceleration, were classified as

190 three groups, specifically, moderate $\left(1.5-2.5 \mathrm{~m} \cdot \mathrm{s}^{-2}\right)$, high $\left(2.6-3.5 \mathrm{~m} \cdot \mathrm{s}^{-2}\right)$ and maximal

$191\left(>3.5 \mathrm{~m} \cdot \mathrm{s}^{-2}\right)$ and presented as a count of how many efforts an athlete undertook per 192 game. 
197 All movement and variables from the present study were presented as descriptive 198 statistics, mean \pm standard deviation (SD). Hypothesis testing was conducted to 199 determine any main effects for movement profile data between position groups on the 200 offensive and defensive teams. A one-way ANOVA was used to determine positional 201 group main effects. In the event homogeneity of variance assumption was violated, a 202 Welch Robust Test of Equality was used to determine main effects between position 203 groups. For all main effects detected by a one-way ANOVA, post-hoc Bonferroni tests 204 were utilized. Alpha intervals for all hypothesis testing were set at $p<0.05$ as the level 205 of significance for statistical tests. All statistical analyses were performed using the 206 Statistical Package for the Social Sciences (SPSS for Windows, version 14.0; SPSS, 207 Inc., Chicago, IL. USA).

\section{RESULTS}

211 Offense: Significant $(p<0.001)$ main effects from ANOVA testing were reported for all 212 movement profile variables measured in the present study for the offensive position 213 groups (Table 3). From post-hoc analysis of movement profile variables, total distance, 214 moderate-intensity distance, high-intensity distance and sprinting distance covered by 215 the WR position was significantly $(p<0.001)$ greater in comparison to all other offensive 216 position groups, including $\mathrm{RB}, \mathrm{QB}, \mathrm{TE}$, and OL. Low-intensity distance covered by the 217 WR position was also significantly $(p<0.001)$ greater for all offensive position groups 218 apart from QB. The QB position group covered significantly $(p<0.001)$ more low- 
219 intensity distance than RB, TE, and OL positions. Moderate-intensity distances were 220 significantly $(p<0.05)$ greater for RB and QB position groups compared to TE and OL

221 position groups. High-intensity distances were significantly $(p<0.01)$ greater for the RB

222 and TE positions compared to QB and OL positions. Sprinting distances were

223 significantly $(p<0.001)$ greater for RB compared to OL. The average max speed

224 achieved by WR, RB and QB positions was significantly $(p<0.05)$ greater than TE and

225 OL positions, while the average max speed achieved by WR position group was

226 significantly $(p<0.05)$ greater than the RB position group.

228 For all high-intensity movement profile variables, including sprint efforts, moderate-, 229 high-intensity, maximal-intensity acceleration and deceleration efforts, the WR position 230 was involved in significantly $(p<0.01)$ more efforts than any other offensive position 231 group. The QB and RB positions were involved in significantly $(p<0.01)$ more sprint 232 efforts per game compared to TE and OL positions. The TE and OL groups were 233 involved in significantly $(p<0.001)$ more moderate acceleration efforts than the RB and 234 QB positions; however, the OL position group had significantly $(p<0.001)$ less maximal 235 acceleration efforts compared to QB and RB positions. The OL position was also 236 involved in significantly $(p<0.001)$ more moderate deceleration efforts compared to the 237 RB position, while for maximal deceleration efforts the OL position was involved in 238 significantly $(p<0.05)$ less than the RB and QB position groups. 
242 Defense: Significant $(p<0.001)$ main effects from ANOVA testing were reported for all

243 movement profile variables measured in the present study for defensive position groups

244 team (Table 4). Post-hoc analysis of movement profile variables including total distance,

245 moderate-intensity distance, high-intensity distance and sprinting distance covered,

246 revealed that both the DB and LB positions covered significantly $(p<0.05)$ greater

247 distances in all zones than the DE and DT positions during games. The only main effect

248 reported for distance covered between the DB and LB position groups was for low-

249 intensity distance covered, with the DB position covering significantly $(p<0.05)$ more

250 than the LB position group. The DB position had the highest average max speed which

251 was significantly $(p<0.05)$ greater than all other defensive positions. The average max

252 speed of the LB position group was significantly $(p<0.05)$ greater than DE and DT

253 positions, although significantly $(p<0.05)$ less than DB. The DE position average max

254 speed was significantly $(p<0.05)$ greater than the DT position, and significantly $(p<$

255 0.05) less than DB and LB positions.

257 The DB position group was involved in significantly $(p<0.05)$ more sprint efforts,

258 moderate-, high-, and maximal-intensity acceleration and deceleration efforts, than the

259 DE and DT positions groups. Apart from moderate acceleration and deceleration efforts

260 and high-intensity deceleration efforts, the DB position group was involved in

261 significantly $(p<0.05)$ more high-intensity movements than the LB position group. The

262 LB position group was involved in significantly $(p<0.05)$ more sprint efforts, high- and

263 maximal-intensity acceleration and deceleration efforts than the DE and DT positions. 
264 Lastly, the DE position group was involved in significantly $(p<0.05)$ more high-intensity 265 acceleration efforts than the DT position group.

\section{DISCUSSION}

271 The present study examined the competitive physiological movement demands of

272 NCAA Division I college football players using portable GPS technology during games,

273 and assessed positional groups within offensive and defensive teams, to determine if a

274 player's physiological requirements during games are influenced by playing position.

275 The results of the present study provide novel insight into the competitive demands 276 experienced by NCAA Division I college football players, and provide scope for the

277 design of position-specific and game-specific physical conditioning strategies for 278 coaches seeking to optimize training for the demands of competition. The results 279 confirm our hypothesis that significant differences in movement profiles accompanying 280 NCAA Division I college football games exist between playing positions. The most 281 notable finding for physical characteristics of games in both offensive and defensive 282 teams were the movement profiles of the WR, DB, and LB positions, with athletes in 283 these three position groups covering more total distance at higher intensities compared 284 to all other positions on their respective offensive and defensive teams. 
286

287

288

289

290

291

292

293

294

295

296

297

298

299

300

301

302

303

304

305

306

307

The total distance covered by athletes in team-sport competition such as American football, may be considered an overall reflection of running volume. The present study found a significant $(p<0.001)$ difference in total distance traveled between position groups within both the offensive and defensive teams. The WR position group covered more total distance per game than all other offensive groups. Similarly on defense, the DB and LB position groups covered greater total distance than the DT and DE position groups. The finding of the present study that the WR, DB, and LB position groups covered greater total distance, is consistent with the work of DeMartini et. al. (8) that found significant differences in distance traveled between linemen $(2573 \pm 489 \mathrm{~m})$ and non-lineman $(3532 \pm 943 \mathrm{~m}$ ) during pre-season training. However, the present study evaluated game data over the course of twelve games compared to DeMartini et. al. (8) who evaluated data obtained during pre-season training in the heat. The absence of published research in relation to the demands of NCAA Division I football games make comparisons with others problematic. Despite the absence of comparable studies, the present results indicate that the total distance covered for both linemen $(3314.0 \mathrm{~m})$ and non-linemen (4141.3 m) during games are greater than those data reported by

DeMartini et. al. (8). From an observational perspective, results from the present study are potentially due to the distance away from the line of scrimmage where the WR, DB and LB position groups started plays. Beginning play further from the line of scrimmage gives athletes a larger area for movement, providing an increased movement requirements during plays and further distances to travel between plays to huddle for brief tactical discussion related to subsequent play. Given WR, DB and LB covered 
greater total running distance throughout games than their offensive and defensive teammates, it is reasonable to suggest athletes in these positions may require modified running volumes in training to support recovery and adequately prepare them for the physical demands of subsequent competition.

In addition to differences in total distance covered by WR, DB, and LB, the present study found significant $(p<0.05)$ differences in moderate-intensity, high-intensity, and sprint distances covered by WR, DB, and LB compared to all other positions on their respective teams. The RB and TE covered significantly $(p<0.05)$ more high-intensity distance than OL. Similar observations in American football training were made by Demartini et. al. (8) who reported non-linemen covering significantly $(p<0.001)$ more high-intensity $\left(>16.0 \mathrm{~km} \cdot \mathrm{h}^{-1}\right)$ distance for position drills, team drills, and total practice time than linemen in pre-season training. Positional differences observed in the present study may be attributed to the position-specific requirements of games. Tactically, the primary responsibility of $\mathrm{OL}$ is to block defensive players, preventing opponents from tackling their own team's ball carrier. These movements are associated with short bursts of acceleration, deceleration, and change of direction, which most frequently occur within a few yards of the line of scrimmage, thereby limiting the distance traveled per play. Players in the DT and DE position groups characteristically accelerate short distances and perform rapid change of direction movements before engaging the opposing OL, followed by pursuing the ball carrier. The position-specific requirements of the OL, DT, and DE positions, requiring a static play initiation posture at or near the line of scrimmage at commencement of each play followed by contact with an opponent 
331 positioned approximately one meter apart, likely influences subsequent running

332 distances. These distances are less than that covered by other positions on the offensive and defensive teams that require players to travel greater distances prior to engaging an opponent. The differences in high-intensity distance covered by TE and $\mathrm{RB}$, compared to $\mathrm{OL}$, may be attributed to the more diverse requirements of these position groups, including blocking, running with the ball, and releasing on pass routes. The WR position group is required to repeatedly run passing routes at high velocities throughout the course of games, consequently accounting for significantly greater highintensity distance and significantly more sprint efforts when compared to all other offensive positions. The DB position group is primarily responsible for defending WR on passing routes, however they also provide secondary support on running plays. As the last line of defense, the DB position is often responsible to make tackles on long running or passing plays, which is indicated in the current study with greater high-intensity distance and more sprint efforts of DB when compared to all other defensive positions.

In addition to the distance covered during play, the WR and DB cover more distance between plays as they are required to jog back to the line of scrimmage at the conclusion of plays, which may be a distance $20-30 \mathrm{~m}$ to either huddle or re-assume their alignment for subsequent play, whereas OL, DT, and DE characteristically walk short distances during recovery between plays (26). The LB position is required to defend running plays in addition to covering $\mathrm{WR}, \mathrm{RB}$ and TE on passing plays which may account for similar movement characteristics to the DB position. The results of the present study highlight the unique movement demands of WR, DB and LB position 
354 groups in comparison to other positions on their respective offensive and defensive 355 teams, and is potentially related to their proximity to the line of scrimmage at the 356 initiation of play. Young et. al. (32) reported greater running distance covered at high 357 speed, along with moderate and high accelerations and decelerations to be associated 358 with markers of muscle damage in collision team-sport players, and consequently, the 359 monitoring and prudent adjustment of weekly training loads of the WR, DB and LB 360 positon groups specifically, may reduce the likelihood of subsequent performance 361 decrements associated with fatigue.

363 Research $(1,21,24)$ in team-sports utilizing portable GPS technology indicate positional differences in movement characteristics during competition. No previous studies have 365 reported the movement demands of NCAA Division I football competition, consequently 366 a lack of understanding exists regarding the demands of American football games.

367 Investigations in team sports similar to American football, including rugby league, rugby 368 union, and Australian rules football, indicate significant differences exist in high-intensity movements including acceleration and deceleration efforts $(28,32)$, and maximal speed

$370(5,24)$ between position groups. The present study found significant differences in 371 maximal running speeds and maximal acceleration and deceleration efforts recorded 372 from offensive position groups. The average max speed of WR position was 373 significantly $(p<0.05)$ greater than all other offensive positions except QB. The RB and 374 QB position groups average max speed was significantly $(p<0.05)$ greater than that of 375 both the TE and OL position groups. The WR group had significantly $(p<0.05)$ more 376 sprint, maximal acceleration, and maximal deceleration efforts than all other offensive 
377 position groups, presumably do to repeated route running requiring sprinting and

378 frequent changes of direction.

380 Defensively, there were no significant differences between total, moderate-, or high381 intensity distance covered between DB and LB position groups, however, significant ( $p$ $382<0.05)$ differences were indicated for average max speed, sprint, maximal acceleration, 383 and maximal deceleration efforts. The DB group had significantly $(p<0.05)$ more 384 sprint, maximal acceleration, and maximal deceleration efforts than all other defensive 385 positions, highlighting the specific high-intensity running requirements of this position 386 during defensive play. The LB position group demonstrated significantly $(p<0.05)$ 387 greater average max speeds, sprint, maximal acceleration, and maximal deceleration 388 efforts than the DE and DT groups. Similar research (8) has not quantified high389 intensity movement characteristics of individual position groups, making comparisons 390 with the present study difficult.

392 The significant differences between the DB group when compared to the defense as a 393 whole, and the LB compared to DT and DE, highlight three distinct running profiles for 394 the defensive team, requiring different forms of training to achieve optimal development. 395 The starting positions upon commencement of each play for the DB and LB groups 396 afford larger areas to achieve higher max speeds, while the positional requirements of 397 defending pass routes and pursuing ball carriers result in greater changes of direction 398 for the DB and LB groups. The WR and DB position groups achieved significantly 
399 greater max speeds, sprint efforts, and maximal acceleration and deceleration efforts 400 than their respective offensive and defensive counterparts throughout the course of 401 games, indicating the need for positional specificity in speed training for NCAA Division I 402 football players.

404 The results of the present study provide novel insight into position-specific physical 405 demands of NCAA Division I football games and provide physical performance staff with 406 quantified information, which can potentially be used to replicate the physical demands

407 of games in training. The present study demonstrated appreciable differences in the 408 positional movement demands of NCAA Division I college football games, emphasizing

409 the need for position-specific training to adequately prepare players for the rigors of 410 competition.

PRACTICAL APPLICATIONS

414 The present study provided a novel analysis of the movement demands associated with 415 NCAA Division I college football games. The results indicated significant differences in 416 total running volume and high-intensity movement demands, most notably for the WR, 417 DB, and LB position groups. Higher overall running loads were experienced for these 418 three position groups, while greater high-intensity movement demands were required of 419 the WR and DB groups. Data from the present study augments our understanding of the competitive demands experienced by NCAA Division I college football players, and 
421 provides scope for the design of position-specific and game-specific physical

422 conditioning strategies for coaches seeking to optimize training for the demands of

423 competition.

425 Data from the present study support the use of position-specific training in the 426 preparation of NCAA Division I college football players for competitive games.

427 Maximizing performance and limiting the effects of fatigue are critical challenges for 428 performance coaches, and as such, accounting for the physical demands associated 429 with weekly training and games is imperative. Modifying weekly training loads of 430 individuals within position groups involved in greater high-speed running volumes and a 431 higher number of acceleration and deceleration efforts may mitigate fatigue, accelerate 432 recovery, and improve subsequent performance. The WR, DB, and LB position groups 433 are exposed to greater running volumes, faster running velocities, and a higher number 434 of acceleration and deceleration efforts in games compared to their offensive and 435 defensive counterparts, and may benefit from carefully monitored and individualized 436 training load prescriptions throughout the week. Additionally, while RB and TE groups 437 do not accrue the total distances during games of the WR group, they are exposed to 438 greater running volumes than the $\mathrm{OL}$, warranting individualized training load 439 prescriptions governed by the physical demands of competition. Clearly, performance 440 coaches seeking to optimize physical performance characteristics associated with 441 competition must differentiate training programs based upon position-specific movement 442 demands. 
444 Data obtained from the present study provide a better understanding of the demands of

445 NCAA Division I football and provide a foundation from which to implement a systematic

446 approach to the development of individual and position-specific training programs.

447 Future studies should examine how coaches seeking to enhance competitive

448 performance, can manipulate individual and position-specific training programs to

449 mitigate fatigue, enhance recovery, and optimize game-day performance.

451 ACKNOWLEDGEMENTS

453 No grant aid or manufacturer's aid was received in conjunction with the present study, 454 and no conflicts of interest are declared. The results of this study do not constitute 455 endorsement of the product by the authors of the National Strength and Conditioning 456 Association.

\section{REFERENCES}

1. Austin, DJ and Kelly, SJ. Positional differences in professional Rugby League match play through the use of global positioning systems. J Strength Cond Res 27: 14-19, 2013.

462 2. Austin, DJ, and Kelly, SJ. Professional Rugby League positional match-play analysis 463 through the use of global positioning system. J Strength Cond Res 28: 187-193, 2013. 
465 3. Barbero-Álvarez, JC, Coutts, A, Granda, J, Barbero-Álvarez, V, and Castagna, C. 466 The validity and reliability of a global positioning satellite system device to assess 467 speed and repeated sprint ability (rsa) in athletes. J Sci Med Sport 13: 232-235, 4682010.

469 4. Boyd, LJ, Ball, K, and Aughey, RJ. Quantifying external load in Australian Football 470 matches and training using accelerometers. Int J Sports Physiol Perform 8: 44-51, 4712013.

472 5. Cahill, N, Lamb, K, Worsfold, P, Headey, R, and Murray, S. The movement characteristics of English Premiership rugby union players. J Sports Sci 31: 229-237,

474 2013.

475

6. Coutts, AJ, and Duffield, R. Validity and reliability of GPS devices for measuring movement demands of team sports. J Sci Med Sport 13: 133-135, 2010.

7. Cunniffe,B, Poctor, W, Baker, JS, and Davies, B. An evaluation of the physiological demands of Elite Rugby Union using global positioning system tracking software. $J$ Strength Cond Res 23: 1195-1203, 2009.

8. DeMartini, JK, Martschinske, JL, Casa, DJ, Lopez, RM, Ganio, MS, Walz, SM, and Coris, EE. Physical demands of National Collegiate Athletic Association division I football players during preseason training in the heat. J Strength Cond Res 25: 2935-2943, 2011.

9. Edgecomb, SJ and Norton, KI. Comparison of global positioning and computerbased tracking systems for measuring player movement distance during Australian 486 football. J Sci Med Sport 9: 25-32, 2006. 
10. Gabbett, TJ, Jenkins, DG, and Abernethy, B. Physical demands of professional rugby league training and competition using microtechnology. J Sci Med Sport 15: 80-86, 2011.

11. Granatelli, G, Gabbett, TJ, Briotti, G, Padulo, J, Buglione, A, D'Ottavio, S, and Ruscello, BM. Match analysis and temporal patterns of fatigue in Rugby Sevens. $J$ Strength Cond Res 28: 728-734, 2014.

12. Iosia, MF, and Bishop, PA. Analysis of exercise-to-rest ratios during division ia televised football competition. J Strength Cond Res 22: 332-340, 2008.

13. Johnston, RD, Gabbett, TJ, and Jenkins, DG. Applied sports science of Rugby League. Sports Med 44: 1087-1100, 2014.

14. Johnston, RD, Watsford, ML, Kelly, SJ, Pine, MJ, and Spurrs, RW. Validity and interunit reliability of $10 \mathrm{hz}$ and $15 \mathrm{hz}$ units for assessing athlete movement demands. J Strength Cond Res 28: 1649-1655, 2014.

15. Johnston, RD, Watsord, ML, Pine, MJ, Spurrs, RW, Murphy, AJ, and Pruyn, EC. The validity and reliability of 5-hz global positioning system units to measure team sport movement demands. J Strength Cond Res 26: 758-765, 2012.

16. Kraemer, WJ and Gotschalk, LA. Physiology of American football. In: Exercise and Sport Science. W.E. Garrett and D.T. Kirkendall, eds. Philadelphia: Lippincott, Williams and Wilkins, 2000. pp. 795-813.

17. Krasnoff, JB, Kohn, MA, Choy, FKK, Doyle, J, Johansen, K, and Painter, PL. Interunit and intraunit reliability of the RT3 triaxial accelerometer. J Phys Act Health 5: 527-538, 2008. 
18. Loader, J, Montgomery, P, Williams, MD, Lorenzen, C, and Kemp, JG. Classifying training drills based on movement demands in Australian Football. Int J of Sports Sci Coach 7: 57-67, 2012.

19. McLellan, CP and Lovell, DI. Neuromuscular responses to impact and collision during elite Rugby League match play. J Strength Cond Res 26: 1431-1440, 2012.

20. McLellan, CP and Lovell, DI. Performance analysis of professional, semiprofessional, and junior elite Rugby League match-play using global positioning systems. J Strength Cond Res 27: 3266-3274, 2013.

21. McLellan, CP, Coad, S, Marsh, D, and Lieschke, M. Performance analysis of Super15 Rugby match-play using portable micro-technology. J Athl Enhanc 2:5, 2013.

22. McLellan, CP, Lovell, DI, and Gass, GC. Biochemical and endocrine responses to impact and collision during elite Rugby League match play. J Strength Cond Res 25: 1553-1562, 2011.

23. McLellan, CP, Lovell, DI, and Gass, GC. Creatine kinase and endocrine responses of elite players pre, during, and post Rugby League match play. J Strength Cond Res 24: 2908-2919, 2010.

24. McLellan, CP, Lovell, DI, and Gass, GC. Performance analysis of elite Rugby League match play using global positioning systems. J Strength Cond Res 25: 17031710, 2011.

25. Petersen, C, Pyne, D, Portus, M, and Dawson, B. Validity and reliability of gps units to monitor cricket-specific movement patterns. Int J Sports Physiol Perform 2: 381393, 2009. 
531 26. Rhea, MR, Hunter, RL, and Hunter, TJ. Competition modeling of American football:

532 observational data and implications for high school, collegiate, and professional

533 player conditioning. J Strength Cond Res 20: 58-61, 2006.

534 27. Robbins, DW, and Young, WB. Positional relationships between various sprint and 535 jump abilities in elite American football players. J Strength Cond Res 26: 388-397, 5362012.

537 28. Sirotic, AC, Knowles, H, Catterick, C, and Coutts, AJ. Positional match demands of 538 professional Rugby League competition. J Strength Cond Res 25: 3076-3087, 2011.

539 29. Sullivan, C, Bilsborough, JC, Cianciosi, M, Hocking, J, Cordy, J, and Coutts, AJ.

540 Match score affects activity profile and skill performance in professional Australian

$541 \quad$ Football Players. J Sci Med Sport 17: 326-331, 2014.

542 30. Varley, MC, Fairweather, IH, and Aughey, RJ. Validity and reliability of GPS for 543 measuring instantaneous velocity during acceleration, deceleration, and constant $544 \quad$ motion. J Sports Sci 30: 121-127, 2012.

545 31. Wisbey, B, Montgomery, PG, Pyne, DB, and Rattray, B. Quantifying movement 546 demands of AFL football using GPS tracking. J Sci Med Sport 13: 531-536, 2010. 547 32. Young, WB, Hepner, J, and Robbins, DW. Movement demands in Australian Rules 548 football as indicators of muscle damage. J Strength Cond Res 26: 492-496, 2012. 